\title{
Article \\ Pyrrolizidine Alkaloids Induce Cell Death in Human HepaRG Cells in a Structure-Dependent Manner
}

\author{
Josephin Glück ${ }^{1}$, Julia Waizenegger ${ }^{1,2}$, Albert Braeuning ${ }^{1}$ and Stefanie Hessel-Pras ${ }^{1, *}$ \\ 1 Department of Food Safety, German Federal Institute for Risk Assessment, Max-Dohrn-Str. 8-10, \\ 10589 Berlin, Germany; josephin.glueck@bfr.bund.de (J.G.); julia.waizenegger@web.de (J.W.); \\ albert.braeuning@bfr.bund.de (A.B.) \\ 2 German Nutrition Society, Godesberger Allee 18, 53175 Bonn, Germany \\ * Correspondence: stefanie.hessel-pras@bfr.bund.de; Tel.: +49-30-18412-25203
}

Citation: Glück, J.; Waizenegger, J.; Braeuning, A.; Hessel-Pras, S. Pyrrolizidine Alkaloids Induce Cell Death in Human HepaRG Cells in a Structure-Dependent Manner. Int. J. Mol. Sci. 2021, 22, 202. https:// dx.doi.org/10.3390/ijms22010202

Received: 27 November 2020 Accepted: 23 December 2020 Published: 28 December 2020

Publisher's Note: MDPI stays neutral with regard to jurisdictional claims in published maps and institutional affiliations.

Copyright: () 2020 by the authors. Licensee MDPI, Basel, Switzerland. This article is an open access article distributed under the terms and conditions of the Creative Commons Attribution (CC BY) license (https: / / creativecommons.org/ licenses/by/4.0/)

\begin{abstract}
Pyrrolizidine alkaloids (PAs) are a group of secondary metabolites produced in various plant species as a defense mechanism against herbivores. PAs consist of a necine base, which is esterified with one or two necine acids. Humans are exposed to PAs by consumption of contaminated food. PA intoxication in humans causes acute and chronic hepatotoxicity. It is considered that enzymatic PA toxification in hepatocytes is structure-dependent. In this study, we aimed to elucidate the induction of PA-induced cell death associated with apoptosis activation. Therefore, 22 structurally different PAs were analyzed concerning the disturbance of cell viability in the metabolically competent human hepatoma cell line HepaRG. The chosen PAs represent the main necine base structures and the different esterification types. Open-chained and cyclic heliotridine- and retronecine-type diesters induced strong cytotoxic effects, while treatment of HepaRG with monoesters did not affect cell viability. For more detailed investigation of apoptosis induction, comprising caspase activation and gene expression analysis, 14 PA representatives were selected. The proapoptotic effects were in line with the potency observed in cell viability studies. In vitro data point towards a strong structure-activity relationship whose effectiveness needs to be investigated in vivo and can then be the basis for a structure-associated risk assessment.
\end{abstract}

Keywords: pyrrolizidine alkaloids; structure dependency; hepatotoxicity; apoptosis

\section{Introduction}

Pyrrolizidine alkaloids (PAs) are a group of secondary metabolites occurring in a wide variety of plants. These natural toxins are produced and stored in plants to protect them from herbivores. More than 660 PAs and their respective $N$-oxides are known and about half of them exhibit hepatotoxic effects [1]. PAs can be found in more than 6000 plant species, especially in the Boraginaceae, Asteraceae and Fabaceae families, representing approximately $3 \%$ of the world's flowering plants [2-5].

Due to their widespread distribution and their genotoxic and hepatotoxic properties, PA-containing plants are among the most common poisonous plants that may affect humans, wildlife and livestock [6]. Exposure of humans and livestock to PAs via the consumption of contaminated food and feed may lead to acute and chronic liver damage comprising the induction of hepatic sinusoidal obstruction syndrome, hepatomegaly, ascites and liver hardening, as well as hepatic necrosis, fibrosis and cirrhosis. In 2013, the German Federal Institute for Risk Assessment identified tea and honey as the main sources for human PA exposure in Western countries. Additionally, the consumption of contaminated herbs, flour or grains and herbal medicines can lead to the intake of substantial quantities [1,7-11].

Chemically, PAs consist of a necine base (1-hydroxymethylpyrrolizidine) that can be esterified at the $\mathrm{OH}$-groups at ring positions C-7 and/or C-9 with aliphatic mono- or dicarboxylic acids (necine acids). The group of PAs can be subdivided according to their 
necine base into the retronecine, heliotridine, otonecine and platynecine types and further by their grade of esterification into free bases, monoesters and cyclic or open-chained diesters. The combination of different necine bases and acids leads to a huge variety of structurally different PAs as summarized in Table 1.

Table 1. Overview of different pyrrolizidine alkaloids (PAs) and their structural characteristics. For cytotoxicity studies, all listed esters and the free bases heliotridine and retronecine were used. The underlined PAs represent the reduced test set for all following experiments.

Cyclic Diesters
Platynecine (7R)

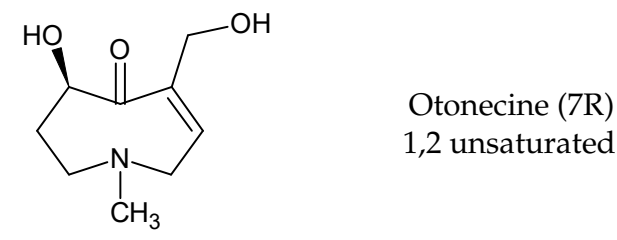

Senkirkine

PAs are stored in plants predominantly in their non-toxic $N$-oxidized form but may be reduced during the digestion process by gut bacteria [12,13]. After intestinal uptake, the PA parent compound can be metabolically activated by cytochrome P450 (CYP) enzymes mainly of the $3 \mathrm{~A}$ and $2 \mathrm{~B}$ subfamilies in the parenchymal cells of the liver [14]. The generation of highly reactive pyrrole esters leads to the formation of protein and DNA adducts $[15,16]$. PAs lacking the double bond in the C-1/C-2 position are considered nonhepatotoxic due to the lack of the structural characteristic needed for bioactivation $[17,18]$.

In addition, numerous in vitro studies indicate that monoesters have a significantly lower toxicity than diesters. According to the current state of knowledge, there is still no scientific consensus with regard to the description of structure-effect relationships of PAs. Studies by Merz and Schrenk (2016), who introduced interim relative potency factors based on the latest in vitro and in vivo data, as well as studies from Louisse et al. (2019) and Allemang et al. (2018), who compared cytotoxic and genotoxic effects of several PAs in vitro, constitute a good starting point for further evaluation of relative toxicity potencies [19-21]. Nevertheless, there are still many uncertainties about how different PAs act at the molecular level. For example, it is not known whether a grouping of PAs according to their cytotoxic effects can be transferred to other endpoints in the same way, or whether the potencies of different PAs vary between several endpoints. Therefore, we focused on the effects of a set of structurally different PAs on the viability of HepaRG cells and the induction of apoptosis. Based on previous studies on the induction of apoptotic 
effects with four structurally different PAs, Waizenegger et al. (2018) stated that the toxicity of a PA seems to be dependent on the PA's necine base. The strongest effects were induced by the cyclic diester senecionine and the open-chained diester echimidine, both belonging to the retronecine-type PAs [22]. To verify this hypothesis, a more extensive set of 22 PAs (Figure 1) was used to investigate the effects in terms of structural properties on the induction of apoptosis in human HepaRG cells.

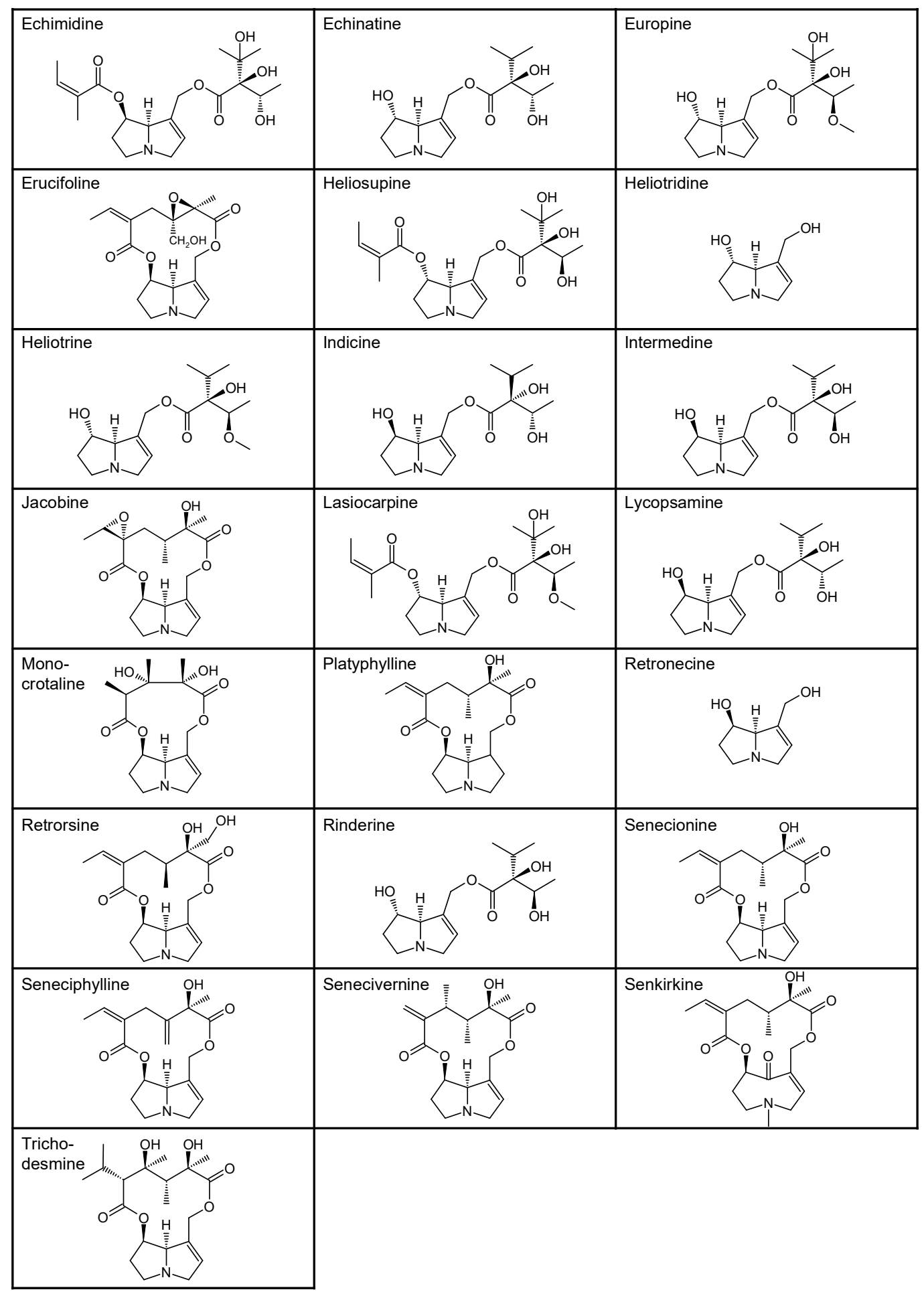

Figure 1. Structures of the 22 pyrrolizidine alkaloids used in this study. 


\section{Results}

\subsection{PA-Induced Cytotoxic Effects in HepaRG}

As a first step, the structure-dependent effects of 22 different PAs on the cell viability of HepaRG cells were analyzed using the MTT cell viability assay (Figure 2). Metabolically competent HepaRG cells were exposed to PAs in concentrations from 0.1 to $250 \mu \mathrm{M}$ for $24 \mathrm{~h}$. Huge differences in the effects on cell viability between the individual PAs can be seen clearly, as shown on the heat map in Figure 2. The monoesters intermedine, indicine, lycopsamine, rinderine, echinatine and europine, as well as the free bases heliotridine and retronecine, affected the viability of HepaRG cells to no or only to a very little extent (remaining cell viability $>90 \%$ ). Open-chained or cyclic diesters induced medium (cell viability between $90 \%$ and $70 \%$ ) to strong cytotoxicity (cell viability < $70 \%$ ). Platyphylline, considered to be not toxic, showed a weak but statistically not significant decrease in cell viability.

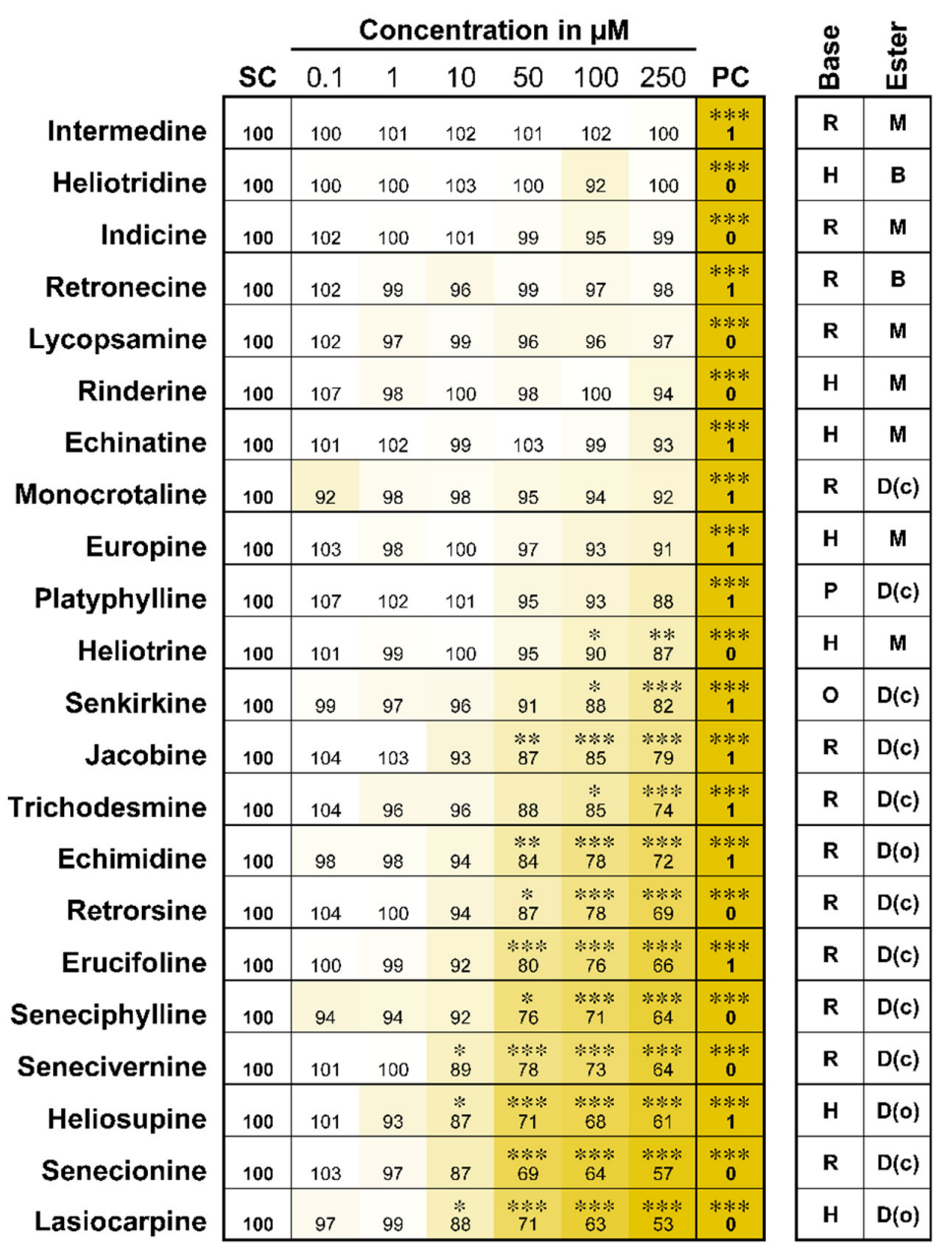

Figure 2. Decrease in viability of HepaRG cells after $24 \mathrm{~h}$ of PA treatment. Cell viability was measured by the MTT assay. Triton X-100 (0.05\%) was used as positive control (PC); the solvent control (SC) contained $2.5 \%$ ACN and $0.5 \%$ DMSO. Data are shown as means of at least three independent replicates normalized to the solvent control. Numbers show the cell viability in percent of the solvent control. The stronger the decrease of cell viability the darker is the yellow coloration in the figure. Values and standard deviations can be found in the Supplemental Material. Statistics: ${ }^{*} p<0.05$, ${ }^{* *} p<0.005,{ }^{* * *} p<0.001$ (one-way ANOVA followed by Dunnett's post hoc test versus the solvent control). Abbreviations for structural characteristics: retronecine $(\mathrm{R})$, heliotrine $(\mathrm{H})$, otonecine $(\mathrm{O})$ or platynecine $(\mathrm{P})$ type; free base $(\mathrm{B})$, monoester $(\mathrm{M})$, open-chained diester $(\mathrm{D}(\mathrm{o}))$ or cyclic diester $(\mathrm{D}(\mathrm{c}))$. 


\subsection{Caspase Induction}

The proteolytic cleavage and thereby activation of caspases is a key event in apoptosis induction. Therefore, the effect of PA exposure on the cleavage of the initiator caspases 8 and 9 and the effector caspases 3 and 7 was analyzed via the fluorescence of specific caspase substrates. Since caspase activation is an early event in apoptosis induction, an incubation time of $6 \mathrm{~h}$ was chosen according to the previous results of Waizenegger et al. (2018) [22]. In addition, PA concentrations in a range that lead to weak cytotoxic effects of the most cytotoxic PAs $(5,21,35 \mu \mathrm{M})$ were selected to reflect the beginning of apoptosis induction. The initial cell viability screening approach comprised a high number of retronecine-type cyclic diesters and heliotridine-type monoesters. Due to the clear structure-dependent grouping of the PAs in the cell viability assay, the PA test set was now reduced to 14 structurally different PAs representatives, comprising a maximum of three members of the same structural group (underlined in Table 1).

In Figure 3 the cleavage of caspase substrates is depicted as a heat map. In the figure, the PAs are sorted according to their cytotoxic activity (cp. Figure 2). While no caspase activation by the less cytotoxic PAs was detected, a concentration-dependent, up to 5.9-fold increase in caspase 3/7 activity was determined for the more toxic PAs echimidine, seneciphylline, heliosupine, senecionine and lasiocarpine. For caspases 8 and 9 , the increase in activity was weaker (up to 1.3- and 2.0-fold) and therefore statistically not significant, even though again the more cytotoxic substances tended to induce a stronger activation of caspases.
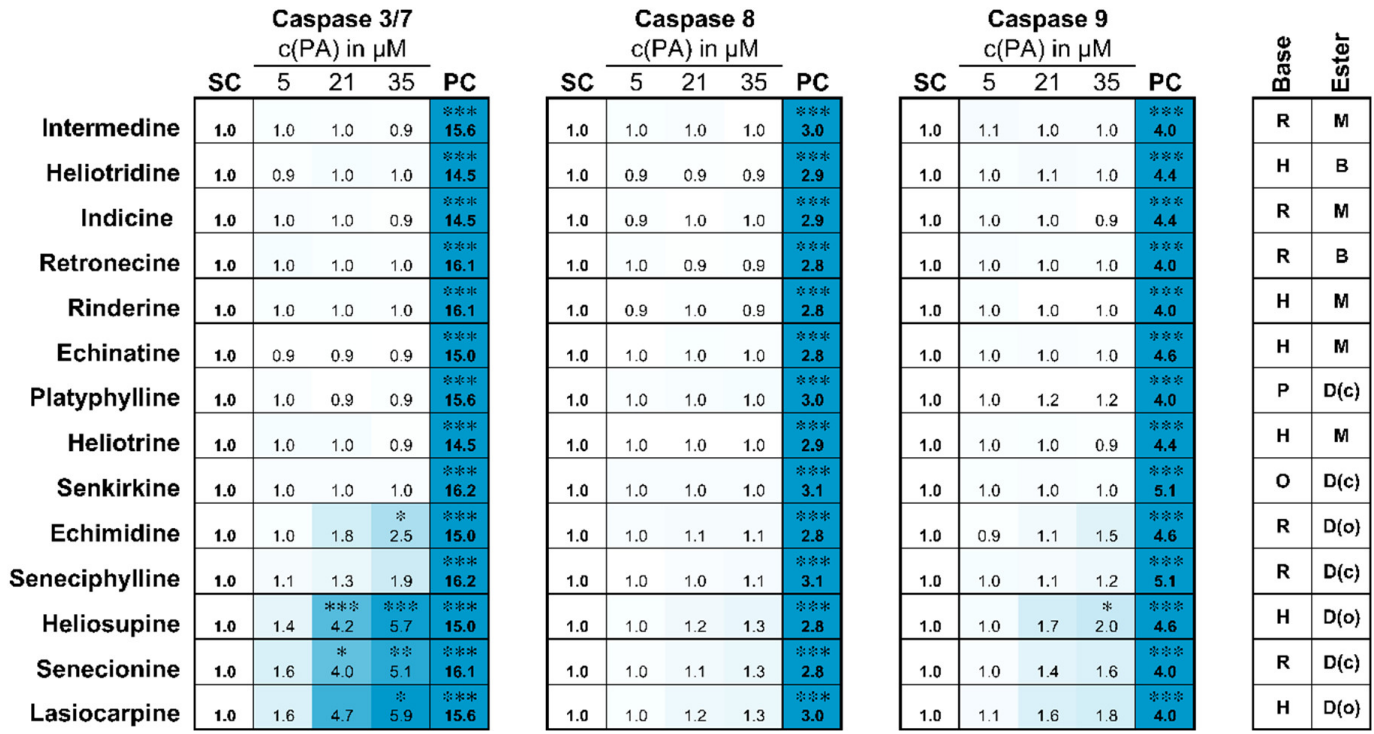

Figure 3. Activation of caspases 3/7, 8 and 9 in HepaRG cells after PA treatment for $6 \mathrm{~h}$. Differentiated HepaRG cells were treated with 5,21 and $35 \mu \mathrm{M}$ of each PA for $6 \mathrm{~h}$. Caspase activities were measured in cell lysates by detecting the cleavage of the specific fluorogenic substrates Ac-DEVD-AFC (caspase 3/7), Ac-IETD-AFC (caspase 8) and Ac-LEHD-AFC (caspase 9). Staurosporine $(5 \mu \mathrm{M})$ was used as positive control (PC) for apoptosis induction; the solvent control (SC) contained $0.35 \%$ $\mathrm{ACN}$ and $0.5 \%$ DMSO. Data are shown as means of three independent replicates. Values indicate the $\mathrm{x}$-fold activation of the caspases normalized to solvent control. The stronger the caspase activity the darker is the blue coloration in the figure. White fields mean no activation of caspases. Mean values and standard deviations can be found in the Supplemental Material. Statistical analysis: ${ }^{*} p<0.05,{ }^{* *} p<0.005,{ }^{* * *} p<0.001$ (one-way ANOVA followed by Dunnett's post hoc analysis versus the solvent control). Abbreviations for structural characteristics: retronecine $(\mathrm{R})$, heliotrine $(\mathrm{H})$, otonecine $(\mathrm{O})$ or platynecine $(\mathrm{P})$ type; free base $(\mathrm{B})$, monoester $(\mathrm{M})$, open-chained diester $(\mathrm{D}(\mathrm{o}))$ or cyclic diester $(\mathrm{D}(\mathrm{c}))$.

\subsection{Expression Analysis of Apoptosis-Related Genes}

Changes in the expression of a set of 16 apoptosis-associated genes were analyzed using quantitative real-time PCR (qPCR). HepaRG cells were treated for $24 \mathrm{~h}$ with the 
test set of 14 PAs in the concentrations also used for analysis of caspase activation (see Section 2.2). The results of gene expression analysis were summarized as a heat map (Figure 4), sorted again by the cytotoxic effects caused by the PAs as shown in Figure 2.

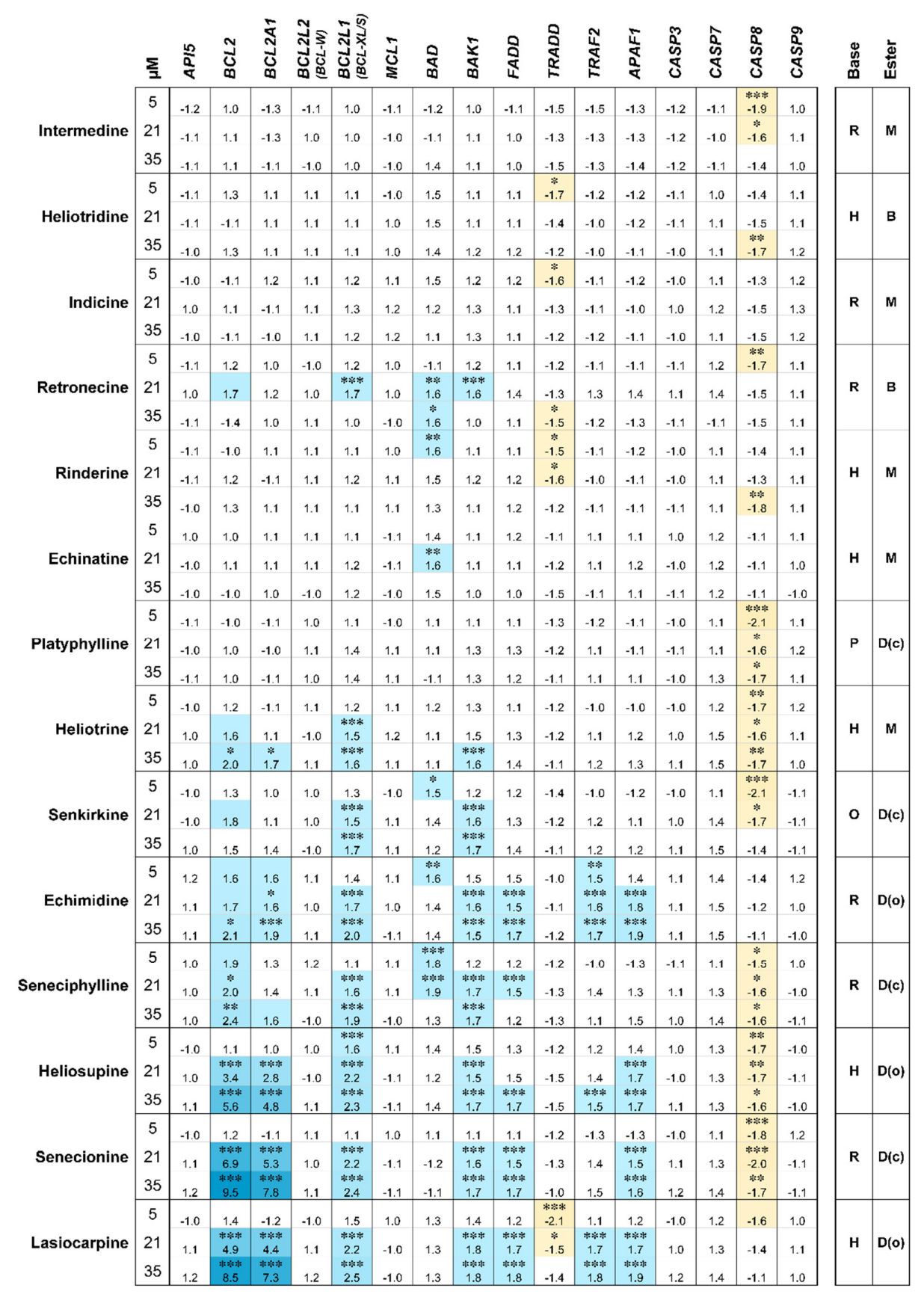

Figure 4. Changes in gene expression levels of apoptosis-associated genes after $24 \mathrm{~h}$ PA exposure of differentiated HepaRG. The results were evaluated according the $2^{-\Delta \Delta \mathrm{Ct}}$ method, normalized to the gene expression of the housekeeping gene GUSB ( $\beta$-glucuronidase) and referred to untreated cells (solvent control, $0.35 \%$ ACN, $0.5 \%$ DMSO). Values are shown as fold change compared to the solvent control and as means of three replicates. The cut-off for gene regulation was set from -1.5 to 1.5. Changes in regulations between -1.5- and 1.5-fold to SC were considered not to be biologically significant. The higher the downregulation of gene expression the darker the yellow, the higher the upregulation the darker the blue coloration. White fields in the figure indicate no gene regulation in the defined fold change cut-off.Mean values and standard deviations are summarized in the Supplemental Material. Statistics: ${ }^{*} p<0.05,{ }^{* *} p<0.005,{ }^{* * *} p<0.001$ (one-way ANOVA followed by Dunnett's post hoc analysis versus the respective solvent control). Structural characteristics: retronecine (R), heliotrine (H), otonecine $(\mathrm{O})$ or platynecine $(\mathrm{P})$ type; free base $(\mathrm{B})$, monoester $(\mathrm{M})$, open-chained diester $(\mathrm{D}(\mathrm{o}))$ or cyclic diester $(\mathrm{D}(\mathrm{c}))$. 
In general, mostly slight alterations were detected at the mRNA level, in line with the fact that apoptosis is predominantly regulated at the protein level by cleavage of target proteins. However, a strong upregulation of the antiapoptotic genes BCL2 and BCL2A1 was detected concentration-dependently for the toxic PAs echimidine, seneciphylline, heliosupine, senecionine and lasiocarpine, reaching up to 9.5-fold (BCL2, senecionine) and 7.8-fold ( $B L C 2 A 1$, senecionine) induction. A slight upregulation of expression was observed for the antiapoptotic gene BCL2L1 and the proapoptotic genes BAK1, FADD and TRAF2. The gene for apoptotic protease activating factor 1 (APAF1) also showed a slight increase in expression after treatment with the more toxic PAs at higher concentrations. For the caspases, with the exception of a slight downregulation of caspase 8 (CASP8), no influence on gene expression was detected. The antiapoptotic gene TRADD, which is, like $C A S P 8$, a part of the extrinsic apoptosis pathway, also showed slight downregulation of expression after treatment of HepaRG with some PAs. However, this effect did not seem to be concentration-dependent and not correlated with the toxicity of the corresponding PAs. The same applies to the very slight regulation of $B A D$. No regulation was observed for the antiapoptotic genes API5, BCL2L2 and MCL1.

\section{Discussion}

Due to their wide distribution and severe toxicity to wildlife, livestock and humans, PAs have long been known as natural plant toxins. Nevertheless, many details about the toxicological modes of action of PAs are still not fully understood. Moreover, PA content and composition differ between plant species, plant organs, geographical origin and growth state. Environmental factors like climate and soil conditions also have a major impact on PA content and composition [23-25]. This additionally aggravates assessment of PA toxicity under real-life conditions.

In risk assessment, it is currently still assumed that all PAs have the same toxicity [26]. However, it is already known that there can be huge differences in toxicity also comprising, for example, phosphorylation of the histone $\mathrm{H} 2 \mathrm{AX}$ and micronucleus formation between the different PAs due to structural reasons [19-21]. It has been shown that monoesters show hardly any toxic effects, while diesters can cause severe liver damage. Furthermore, 1,2-unsaturated PAs such as platyphylline cannot be metabolically activated and therefore no reactive metabolites are produced that form DNA and protein adducts $[17,18]$. PA $\mathrm{N}$-oxides, which are predominantly found in plants, cannot be directly activated either. However, it is possible that these can be reduced to the PA parent substance during the digestion process $[12,13]$. Thus, a risk assessment which considers all 1,2-saturated PAs as a homogenous group with equipotent representatives contains a considerable degree of uncertainty, while an approach based on a structure effect-oriented assessment may yield more precise results. In 2016, Merz and Schrenk took a first step towards standardizing the available data and proposed the introduction of relative potency factors for a number of PAs by combining genotoxic, cytotoxic and acute toxic effects in vivo and in vitro [20]. Based on this grouping of PAs according to their toxic potential, a more refined picture of PA toxicity may be developed through targeted studies on various endpoints.

A whole transcriptome analysis by Luckert et al. in 2015 showed dramatic changes in the expression of a wide variety of genes after $24 \mathrm{~h}$ of exposure of primary human hepatocytes to the four PAs echimidine, heliotrine, senecionine and senkirkine. Softwarebased analyses of the regulated genes indicated that, among others, cellular mechanisms such as cell death, apoptosis and necrosis could be impaired [27]. Other studies have also indicated connections between PA exposure and the induction of apoptosis in the liver for the PAs monocrotaline, clivorine, retrorsine and senecionine [28-32]. Based on these data, we focused on structural differences in the induction of cytotoxicity and especially apoptosis by 22 different PAs. Based on previous studies by Waizenegger et al. and Luckert et al. with only four different PAs, we hypothesized that retronecine-type PAs possess the strongest toxic potential [22,27]. This assumption needs to be refined when considering the results of this study: the necine base appears to play a rather minor role in toxicity, as the 
effects of lasiocarpine and heliosupine (heliotridine type) were largely equivalent to those of senecionine and seneciphylline (retronecine type). Furthermore, we confirmed that PA monoesters and free necine bases induce no to very weak toxicity, while PA diesters have medium to strong toxic potential concerning the induction of cytotoxicity in HepaRG cells.

The grouping of PAs according to their cytotoxic potential was confirmed in the following analysis of caspase activation with a slightly reduced test set. The effector caspases 3 and 7 were substantially activated despite PA concentrations below clearly cytotoxic levels. The activation of the initiator caspases 8 and 9 was less pronounced. PA-induced apoptosis seems to be more likely to occur via the intrinsic signaling pathway (caspase 9-mediated) [28,30,32]. Waizenegger et al. (2018) and Ebmeyer et al. (2019) could nevertheless show that the extrinsic signaling pathway (mediated by Fas via caspase 8) may also be involved. Thus, PA-mediated activation of apoptosis pathways seems to occur via a combination of both extrinsic and intrinsic mechanisms [22,33].

The classification of PAs can also be applied to changes in the expression of apoptosisassociated genes. The effect is particularly evident for the genes encoding the antiapoptotic proteins BCL2 and BCL1A2. The upregulation of the expression of these genes is probably a feedback mechanism of apoptosis induction. The regulation of the apoptotic process mainly takes place at the protein level. The cleavage and degradation of the proteins BCL2 and BCL1A2 decrease the intracellular protein amount and thus their expression is upregulated at the gene level, possibly as part of a feedback mechanism.

Summarizing all the data of this study, the PA with the highest toxic potential are lasiocarpine and heliosupine (both heliotridine-type, open-chained diesters), senecionine and seneciphylline (both retronecine-type, cyclic diesters) and echimidine (retronecinetype, open-chained diesters). Different PA representatives were analyzed in regard to other, more genotoxicity-related endpoints beside apoptosis induction in recent published studies: Louisse et al. detected DNA double-strand break induction by analyzing the phosphorylation of the histone H2AX after exposure of HepaRG cells to 37 different PAs in several concentrations [19]. The micronucleus assay as an endpoint for genotoxicity was performed by Allemang et al. for 15 different PAs and PA N-oxides in HepaRG cells [21]. Rutz et al. (2020) also analyzed 11 PAs with regard to the induction of cytotoxicity and the formation of micronuclei in CYP3A4-overexpressing HepG2 cells, as well as the induction of mutations using the Ames fluctuation test [34]. Gao et al. investigated the cytotoxicity of 10 PAs in primary rat hepatocytes [35]. Lester et al. investigated the formation of DHP-DNA adducts in rat hepatocytes after exposure to nine different PAs [36]. In all the studies mentioned, heliosupine, lasiocarpine, senecionine and seneciphylline, if used, showed very strong toxic effects compared to the effects of other PAs, which fits to the results of this study. The strength of toxicity of echimidine varies between the publications but is nevertheless always in the medium toxicity range. Minor differences between the effects of PAs can most likely be attributed to different test systems (cell lines), solvents or tested endpoints. The quantity and activity of xenobiotic-metabolizing enzymes with their metabolic activation and detoxification capacities, for example, have a direct influence on the toxicity of PAs [37]. This is why differences between the individual cell types and culture conditions are particularly important here. However, despite the differences in the test set-up, only minor deviations in the effects of most PAs are shown between the different studies. Since the effects are largely comparable, the sorting of PAs according to their cytotoxic potential can be considered as reliable for in vitro analyses with cytotoxic or genotoxic endpoints.

Although the results of the studies are broadly similar for many PAs, there are large differences for some PAs such as monocrotaline. For this PA, a clear discrepancy can be seen between the relative potency factors according to Merz and Schrenk [20] and the above-mentioned in vitro studies. The introduction of relative potency factors is therefore controversial and must be considered critically. There is already a large amount of toxicity data on various PAs from studies that have been conducted in vivo or in vitro. Unfortunately, these data can often not be compared directly with each other because different PAs 
were used in different species and/or cell lines, with different incubation times, doses or concentrations, and different endpoints or methods. In addition, the comparison between in vitro and in vivo data is difficult because aspects such as metabolism and uptake in the digestive tract, distribution in the body and excretion are not considered in in vitro studies. Furthermore, metabolic activation in the liver and other organs cannot be mapped 1:1 in vitro either. Even very metabolically competent cell lines often show a deficiency of certain enzymes [38-40].

Previous investigations on the structure dependence of PA toxicity in vitro are not sufficient for use in risk assessment. For a reliable assessment of the risk of PA contamination in food to humans, some gaps need to be closed. In order to refine the classification of PAs according to their toxic potential, it is necessary to examine structure-dependent toxicokinetic properties and toxification reactions in more detail. It is therefore necessary to extend in vitro and in vivo studies to enable a precise derivation of potency factors to refine the assessment of risk of PA exposure to humans.

\section{Materials and Methods}

\subsection{Chemicals}

All PAs used in this study were purchased from Phytoplan Diehm \& Neuberger GmbH (Heidelberg, Germany), except platyphylline which was obtained from BOC Sciences (New York, NY, USA). The purity of all PAs was at least $95 \%$. PAs were stored at $-20{ }^{\circ} \mathrm{C}$ dissolved in $50 \%$ Acetonitrile $(\mathrm{ACN}) / 50 \% \mathrm{H}_{2} \mathrm{O}$ as $5 \mathrm{mM}$ stock solutions. All other chemicals were obtained from Merck (Darmstadt, Germany) or Sigma-Aldrich (Taufkirchen, Germany) in the highest purity available.

\subsection{HepaRG Cell Culture}

The human hepatoma cell line HepaRG was obtained from Biopredic International (Saint-Gregoire, France). After seeding, the cells were cultivated for two weeks in William's Medium E with stable glutamine (PAN Biotech, Aidenbach, Germany) supplemented with $10 \%$ fetal bovine serum (FBS Good forte, PAN-Biotech, Aidenbach, Germany), $100 \mathrm{U} / \mathrm{mL}$ penicillin and $100 \mu \mathrm{g} / \mathrm{mL}$ streptomycin (PAN-Biotech, Aidenbach, Germany), $5 \mu \mathrm{g} / \mathrm{mL}$ human insulin (PAN-Biotech, Aidenbach, Germany) and $50 \mu \mathrm{M}$ hydrocortisone hemisuccinate (Sigma-Aldrich, Taufkirchen, Germany). After this proliferation phase, differentiation was initiated by adding $1.7 \%$ dimethyl sufoxide (DMSO). HepaRG cells were fully differentiated after two weeks. All experiments with HepaRG were performed with differentiated cells seeded at passages 16 to 20 .

\subsection{Cell Viability Assay}

HepaRG cells were seeded at a density of 9000 cells per well in the inner 60 wells of a 96-well plate. After four weeks of cultivation, DMSO and FBS in the cell culture medium were set to $0.5 \%$ and $2 \%$ for $48 \mathrm{~h}$ before incubation, respectively, to optimize the effects on gene expression. The cells were treated with different PA concentrations for $24 \mathrm{~h}$ as indicated in the figures according to Waizenegger et al. (2018) [22]. Due to the low solubility of some PA, concentrations higher than $250 \mu \mathrm{M}$ would lead to cytotoxic concentrations of the solvent ACN. For the cell viability assay, $10 \mu \mathrm{L}$ undiluted MTT (3(4,5-dimethylthiazol-2-yl)-2,5-diphenyltetrazolium bromide) reagent per well was added. After $30 \mathrm{~min}$ of incubation at $37^{\circ} \mathrm{C}$, the supernatant was discarded and the violet formazan crystals were dissolved in $130 \mu \mathrm{L}$ isopropanol with $0.7 \%$ sodium dodecyl sulfate (SDS) per well (30 min, shaking protected from light). Absorption was determined at 570 and $630 \mathrm{~nm}$ (reference wavelength) [41].

\subsection{Total RNA Isolation and Quantitative Real-Time Polymerase Chain Reaction (qPCR)}

To investigate effects of PA treatment on the expression of apoptosis-associated genes, gene expression was analyzed using quantitative reverse transcription real-time PCR. HepaRG cells were seeded in 6-well plates at a density of $0.2 \times 10^{6}$ cells per well. DMSO and 
FBS were set to $0.5 \%$ and $2 \%$ for $48 \mathrm{~h}$ before PA treatment to optimize inducibility of gene expression. The cells were incubated with different PA concentrations for $24 \mathrm{~h}$. Afterwards, cells were washed twice with cold PBS. The RNeasy Mini Kit (Qiagen, Hilden, Germany) was used for total RNA extraction following the manufacturer's instructions including the on-column DNA digestion step. RNA concentration and purity were measured at 260, 280 and $230 \mathrm{~nm}$ on a TecanM200Pro spectrophotometer using a NanoQuant plate. The ratios A260/A280 and A260/ A230 were calculated to detect potential contaminants.

For cDNA synthesis, the High Capacity cDNA Reverse Transcriptase Kit (Applied Biosystems, Foster City, CA, USA) was used as recommended by the manufacturer with 1 $\mu \mathrm{g}$ RNA per reaction. For qPCR analysis $1 \mu \mathrm{L}$ cDNA, $5 \mu \mathrm{L}$ Maxima SYBR Green/ROX qPCR Master Mix (Thermo Fisher Scientific, Waltham, MA, USA) and $300 \mathrm{nM}$ of each primer were used in a total volume of $10 \mu \mathrm{L}$ per reaction. In each PCR run, at least three wells without a template were used as control. The following thermal profile was used: initial denaturation $\left(15 \mathrm{~min}, 95^{\circ} \mathrm{C}\right), 40$ cycles of denaturation $\left(30 \mathrm{~s}, 95^{\circ} \mathrm{C}\right)$ and annealing/elongation $(1 \mathrm{~min}$, $\left.60{ }^{\circ} \mathrm{C}\right)$, final elongation $\left(10 \mathrm{~min}, 60^{\circ} \mathrm{C}\right)$. The specific amplification of a single PCR product was confirmed by the analysis of the recorded dissociation curve. The cDNA amplification of three replicates per sample was detected at the 7900HT Fast Real-Time PCR System (Applied Biosystems, Foster City, CA, USA) in 384-well format. The Ct values were normalized to the housekeeping gene GUSB ( $\beta$-glucuronidase), evaluated according the $2^{-\Delta \Delta C t}$ method [42] and referred to solvent control. The primer sequences are summarized in the supplementary data. GUSB was used as a housekeeping gene with a constitutive expression after validation for the HepaRG cell line and the exposure to PAs.

\subsection{Activation of Caspases}

The caspase activation assay was performed as described previously [22]. Briefly, differentiated HepaRG cells were incubated with PAs for $6 \mathrm{~h}$ in 96-well plates. After treatment, cells were lysed using 50 mM HEPES buffer ( $\mathrm{pH} 7.4$ ) containing $2 \%$ Triton X-100. Specific fluorogenic substates for caspases 3/7 (Ac-DEVD-AFC), caspase 8 (Ac-IETD-AFC) and caspase 9 (Ac-LEHD-AFC) were added to the cells for $1 \mathrm{~h}\left(37^{\circ} \mathrm{C}\right)$. The fluorescence of the cleaved substrates was detected at $\lambda_{\mathrm{ex}}=380$ and $\lambda_{\mathrm{em}}=500 \mathrm{~nm}$ (DEVD and IETD), or at $\lambda_{\mathrm{ex}}=400$ and $\lambda_{\mathrm{em}}=505 \mathrm{~nm}$ (LEHD).

\subsection{Statistical Analysis}

Statistical analysis was performed using Sigmaplot 14.0 software (Systat Software, Erkrath, Germany). To determine statistical differences in a concentration series, oneway analysis of variance (ANOVA) followed by the Dunnett's post hoc test versus the respective solvent control was performed. Statistically significant differences are indicated by ${ }^{*} p<0.05,{ }^{* *} p<0.005,{ }^{* * *} p<0.001$.

Supplementary Materials: Supplementary Materials can be found at https://www.mdpi.com/1422 $-0067 / 22 / 1 / 202 /$ s1.

Author Contributions: J.G. conceptualization, investigation, visualization, writing-original draft. J.W. conceptualization, investigation. A.B. conceptualization, supervision, writing-review and editing. S.H.-P. conceptualization, funding acquisition, project administration, writing-review and editing. All authors have read and agreed to the published version of the manuscript.

Funding: This work was supported by the German Research Foundation (Grant number LA1177/121) and by the German Federal Institute for Risk Assessment (grant number 1322-624).

Data Availability Statement: The data presented in this study are available in the Supplementary Materials which can be found at https://www.mdpi.com/1422-0067/22/1/202/s1.

Acknowledgments: We thank Doreen Stern for her excellent technical support.

Conflicts of Interest: The authors declare no conflict of interest. 


$\begin{array}{ll}\text { Abbreviations } & \\ \text { Ac-DEVD-AFC } & \text { N-Acetyl-Asp-Glu-Val-Asp-7-amido-4-trifluoromethylcoumarin } \\ \text { Ac-IETD-AFC } & \text { N-Acetyl-Ile-Glu-Thr-Asp-7-amido-4-trifluoromethylcoumarin } \\ \text { Ac-LEHD-AFC } & \text { N-Acetyl-Leu-Glu-His-Asp-7-amido-4-trifluoromethylcoumarin } \\ \text { ACN } & \text { Acetonitrile } \\ \text { CYP } & \text { Cytochrome P450-monooxygenases } \\ \text { DHP } & \text { Dehydropyrrolizidine alkaloid } \\ \text { DMSO } & \text { Dimethyl sulfoxide } \\ \text { FBS } & \text { Fetal bovine serum } \\ \text { MTT } & \text { 3-(4,5-Dimethylthiazol-2-yl)-2,5-diphenyltetrazoliumbromid } \\ \text { PA(s) } & \text { Pyrrolizidine alkaloid(s) } \\ \text { (q)PCR } & \text { (quantitative) Polymerase chain reaction } \\ \text { SDS } & \text { Sodium dodecyl sulfate }\end{array}$

\section{References}

1. Edgar, J.A.; Roeder, E.; Molyneux, R.J. Honey from plants containing pyrrolizidine alkaloids: A potential threat to health. J. Agric. Food Chem. 2002, 50, 2719-2730. [CrossRef] [PubMed]

2. Roeder, E. Medicinal plants in Europe containing pyrrolizidine alkaloids. Pharmazie 1995, 50, 83-98. [PubMed]

3. Roeder, E. Medicinal plants in China containing pyrrolizidine alkaloids. Pharmazie 2000, 55, 711-726. [PubMed]

4. Reimann, A.; Nurhayati, N.; Backenkohler, A.; Ober, D. Repeated evolution of the pyrrolizidine alkaloid-mediated defense system in separate angiosperm lineages. Plant Cell 2004, 16, 2772-2784. [CrossRef] [PubMed]

5. Smith, L.W.; Culvenor, C.C. Plant sources of hepatotoxic pyrrolizidine alkaloids. J. Nat. Prod. 1981, 44, 129-152. [CrossRef] [PubMed]

6. Stegelmeier, B.L.; Edgar, J.A.; Colegate, S.M.; Gardner, D.R.; Schoch, T.K.; Coulombe, R.A.; Molyneux, R.J. Pyrrolizidine alkaloid plants, metabolism and toxicity. J. Nat. Toxins 1999, 8, 95-116.

7. Kakar, F.; Akbarian, Z.; Leslie, T.; Mustafa, M.L.; Watson, J.; van Egmond, H.P.; Omar, M.F.; Mofleh, J. An outbreak of hepatic veno-occlusive disease in Western afghanistan associated with exposure to wheat flour contaminated with pyrrolizidine alkaloids. J. Toxicol. 2010, 2010, 313280. [CrossRef]

8. BfR. Pyrrolizidine Alkaloids in Herbal Teas and Teas. German Federal Insitute for Risk Assessment. BfR Opinion. 2013 , pp. 1-31. Available online: https:/ / www.bfr.bund.de/cm/349/pyrrolizidine-alkaloids-in-herbal-teas-and-teas.pdf (accessed on 5 July 2013).

9. Bodi, D.; Ronczka, S.; Gottschalk, C.; Behr, N.; Skibba, A.; Wagner, M.; Lahrssen-Wiederholt, M.; Preiss-Weigert, A.; These, A. Determination of pyrrolizidine alkaloids in tea, herbal drugs and honey. Food Addit. Contam. Part A Chem. Anal. Control Expo. Risk Assess. 2014, 31, 1886-1895. [CrossRef]

10. Mulder, P.P.J.; Lopez, P.; Castellari, M.; Bodi, D.; Ronczka, S.; Preiss-Weigert, A.; These, A. Occurrence of pyrrolizidine alkaloids in animal- and plant-derived food: Results of a survey across Europe. Food Addit Contam Part. A Chem Anal. Control. Expo. Risk Assess. 2018, 35, 118-133. [CrossRef]

11. Tamariz, J.; Burgueño-Tapia, E.; Vázquez, M.A.; Delgado, F. Pyrrolizidine Alkaloids. Alkaloids Chem. Biol. 2018, 80, 1-314. [CrossRef]

12. Yang, M.; Ma, J.; Ruan, J.; Ye, Y.; Fu, P.P.; Lin, G. Intestinal and hepatic biotransformation of pyrrolizidine alkaloid N-oxides to toxic pyrrolizidine alkaloids. Arch. Toxicol. 2019, 93, 2197-2209. [CrossRef] [PubMed]

13. Hartmann, T.; Witte, L. Chapter Four-Chemistry, Biology and Chemoecology of the Pyrrolizidine Alkaloids. In Alkaloids: Chemical and Biological Perspectives; Pelletier, S.W., Ed.; Pergamon: Oxford, UK, 1995; Volume 9, pp. $155-233$.

14. Fu, P.P.; Xia, Q.; Lin, G.; Chou, M.W. Pyrrolizidine alkaloids-genotoxicity, metabolism enzymes, metabolic activation, and mechanisms. Drug Metab. Rev. 2004, 36, 1-55. [CrossRef] [PubMed]

15. Chen, T.; Mei, N.; Fu, P.P. Genotoxicity of pyrrolizidine alkaloids. J. Appl. Toxicol. 2010, 30, 183-196. [CrossRef] [PubMed]

16. Ruan, J.; Yang, M.; Fu, P.; Ye, Y.; Lin, G. Metabolic activation of pyrrolizidine alkaloids: Insights into the structural and enzymatic basis. Chem. Res. Toxicol. 2014, 27, 1030-1039. [CrossRef] [PubMed]

17. Ruan, J.; Liao, C.; Ye, Y.; Lin, G. Lack of metabolic activation and predominant formation of an excreted metabolite of nontoxic platynecine-type pyrrolizidine alkaloids. Chem. Res. Toxicol. 2014, 27, 7-16. [CrossRef] [PubMed]

18. Mattocks, A.R.; White, I.N. Pyrrolic metabolites from non-toxic pyrrolizidine alkaloids. Nat. Neww Biol. 1971, 231, 114-115. [CrossRef]

19. Louisse, J.; Rijkers, D.; Stoopen, G.; Holleboom, W.J.; Delagrange, M.; Molthof, E.; Mulder, P.P.J.; Hoogenboom, R.; Audebert, M.; Peijnenburg, A. Determination of genotoxic potencies of pyrrolizidine alkaloids in HepaRG cells using the $\gamma \mathrm{H} 2 \mathrm{AX}$ assay. Food Chem. Toxicol. 2019, 131, 110532. [CrossRef]

20. Merz, K.H.; Schrenk, D. Interim relative potency factors for the toxicological risk assessment of pyrrolizidine alkaloids in food and herbal medicines. Toxicol. Lett. 2016, 263, 44-57. [CrossRef]

21. Allemang, A.; Mahony, C.; Lester, C.; Pfuhler, S. Relative potency of fifteen pyrrolizidine alkaloids to induce DNA damage as measured by micronucleus induction in HepaRG human liver cells. Food Chem. Toxicol. 2018, 121, 72-81. [CrossRef]

22. Waizenegger, J.; Braeuning, A.; Templin, M.; Lampen, A.; Hessel-Pras, S. Structure-dependent induction of apoptosis by hepatotoxic pyrrolizidine alkaloids in the human hepatoma cell line HepaRG: Single versus repeated exposure. Food Chem. Toxicol. 2018, 114, 215-226. [CrossRef] 
23. O'dowd, D.J.; Edgar, J.A. Seasonal dynamics in the pyrrolizidine alkaloids of Heliotropium europaeum. Aust. J. Ecol. 1989, 14, 95-105. [CrossRef]

24. Kirk, H.; Vrieling, K.; Van Der Meijden, E.; Klinkhamer, P.G.L. Species by Environment Interactions Affect Pyrrolizidine Alkaloid Expression in Senecio jacobaea, Senecio aquaticus, and Their Hybrids. J. Chem. Ecol. 2010, 36, 378-387. [CrossRef] [PubMed]

25. Johnson, A.E.; Molyneux, R.J.; Merrill, G.B. Chemistry of toxic range plants-Variation in pyrrolizidine alkaloid content of senecio, amsinckia, and crotalaria species. J. Agric. Food Chem. 1985, 33, 50-55. [CrossRef]

26. Panel, E.C. Risks for human health related to the presence of pyrrolizidine alkaloids in honey, tea, herbal infusions and food supplements. EFSA J. 2017, 15, e04908.

27. Luckert, C.; Hessel, S.; Lenze, D.; Lampen, A. Disturbance of gene expression in primary human hepatocytes by hepatotoxic pyrrolizidine alkaloids: A whole genome transcriptome analysis. Toxicol. In Vitro 2015, 29, 1669-1682. [CrossRef]

28. Yang, X.; Wang, H.; Ni, H.M.; Xiong, A.; Wang, Z.; Sesaki, H.; Ding, W.X.; Yang, L. Inhibition of Drp1 protects against senecionineinduced mitochondria-mediated apoptosis in primary hepatocytes and in mice. Redox Biol. 2017, 12, 264-273. [CrossRef]

29. Copple, B.L.; Rondelli, C.M.; Maddox, J.F.; Hoglen, N.C.; Ganey, P.E.; Roth, R.A. Modes of Cell Death in Rat Liver after Monocrotaline Exposure. Toxicol. Sci. 2004, 77, 172-182. [CrossRef]

30. Liu, W.; Li, X.; Zhou, B.; Fang, S.; Ho, W.; Chen, H.; Liang, H.; Ye, L.; Tang, J. Differential induction of apoptosis and autophagy by pyrrolizidine alkaloid clivorine in human hepatoma Huh-7.5 cells and its toxic implication. PLoS ONE 2017, 12, e0179379. [CrossRef]

31. Gordon, G.J.; Coleman, W.B.; Grisham, J.W. Bax-mediated apoptosis in the livers of rats after partial hepatectomy in the retrorsine model of hepatocellular injury. Hepatology 2000, 32, 312-320. [CrossRef]

32. Ji, L.; Chen, Y.; Liu, T.; Wang, Z. Involvement of Bcl-xL degradation and mitochondrial-mediated apoptotic pathway in pyrrolizidine alkaloids-induced apoptosis in hepatocytes. Toxicol. Appl. Pharmacol. 2008, 231, 393-400. [CrossRef]

33. Ebmeyer, J.; Franz, L.; Lim, R.; Niemann, B.; Glatt, H.; Braeuning, A.; Lampen, A.; Hessel-Pras, S. Sensitization of Human Liver Cells Toward Fas-Mediated Apoptosis by the Metabolically Activated Pyrrolizidine Alkaloid Lasiocarpine. Mol. Nutr. Food Res. 2019, 63, e1801206. [CrossRef] [PubMed]

34. Rutz, L.; Gao, L.; Kupper, J.H.; Schrenk, D. Structure-dependent genotoxic potencies of selected pyrrolizidine alkaloids in metabolically competent HepG2 cells. Arch. Toxicol. 2020. [CrossRef] [PubMed]

35. Gao, L.; Rutz, L.; Schrenk, D. Structure-dependent hepato-cytotoxic potencies of selected pyrrolizidine alkaloids in primary rat hepatocyte culture. Food Chem. Toxicol. 2020, 135, 110923. [CrossRef] [PubMed]

36. Lester, C.; Troutman, J.; Obringer, C.; Wehmeyer, K.; Stoffolano, P.; Karb, M.; Xu, Y.; Roe, A.; Carr, G.; Blackburn, K.; et al. Intrinsic relative potency of a series of pyrrolizidine alkaloids characterized by rate and extent of metabolism. Food Chem. Toxicol. 2019, 131, 110523. [CrossRef] [PubMed]

37. Ebmeyer, J.; Braeuning, A.; Glatt, H.; These, A.; Hessel-Pras, S.; Lampen, A. Human CYP3A4-mediated toxification of the pyrrolizidine alkaloid lasiocarpine. Food Chem. Toxicol. 2019, 130, 79-88. [CrossRef] [PubMed]

38. Andersson, T.B.; Kanebratt, K.P.; Kenna, J.G. The HepaRG cell line: A unique in vitro tool for understanding drug metabolism and toxicology in human. Expert Opin. Drug Metab. Toxicol. 2012, 8, 909-920. [CrossRef]

39. Sison-Young, R.L.C.; Mitsa, D.; Jenkins, R.E.; Mottram, D.; Alexandre, E.; Richert, L.; Aerts, H.; Weaver, R.J.; Jones, R.P.; Johann, E.; et al. Comparative Proteomic Characterization of 4 Human Liver-Derived Single Cell Culture Models Reveals Significant Variation in the Capacity for Drug Disposition, Bioactivation, and Detoxication. Toxicol. Sci. 2015, 147, 412-424. [CrossRef]

40. Le Vee, M.; Jigorel, E.; Glaise, D.; Gripon, P.; Guguen-Guillouzo, C.; Fardel, O. Functional expression of sinusoidal and canalicular hepatic drug transporters in the differentiated human hepatoma HepaRG cell line. Eur. J. Pharm. Sci. 2006, 28, 109-117. [CrossRef]

41. Mosmann, T. Rapid colorimetric assay for cellular growth and survival: Application to proliferation and cytotoxicity assays. J. Immunol. Methods 1983, 65, 55-63. [CrossRef]

42. Livak, K.J.; Schmittgen, T.D. Analysis of Relative Gene Expression Data Using Real-Time Quantitative PCR and the $2^{-\Delta \Delta C T}$ Method. Methods 2001, 25, 402-408. [CrossRef] 